\title{
A Wearable Patient Healthcare Monitoring System Using IoT and Cloud Computing Based Security
}

\author{
Chitra Selvi $\mathrm{S}^{\mathrm{a}, 1}$ \\ ${ }^{a}$ Professor, Dept of EEE, University College of Engineering, Thirukkuvalai, India
}

\begin{abstract}
Now-a-days, a developing number of individuals at some point of a developing international locations like India forces to seem for brand spanking new answers for the persistent tracking of fitness check-up for stable data. It's emerge as a need to go to hospitals frequently for doctor's consultation, which has Growth to be financially associated and a time ingesting process. To beat this situation, we endorse a design to observe the patient's fitness situations like heartbeat, temperature, ECG and BP and ship the message to guardian the use of GSM. Within the recent improvement of internet of factors (IoT) makes all objects interconnected and cloud been diagnosed due to the fact the subsequent technical revolution and now not secure for patient data. Patient monitoring is one a few of the IoT application to watch the affected person fitness status to collect facts to security for both medical doctor and patients Internet of things makes clinical equipment greater efficient with the aid of allowing actual time tracking of health in security privateness of the patient. Using IoT doctor can continuously monitor the patient's cloud the usage of protection on his smartphone and also the affected person history could be stored on the web server and health practitioner can get entry to the statistics on every occasion wished from anywhere.
\end{abstract}

Keywords. Internet-of-Things, cloud computing, privacy or services

\section{Introduction}

Now-a-days, the medical electronics-sensors (E-sensors) plays a major role in health care centers [9]. The affected person electronics-health monitoring is one many of the important improvements in medical field. Here we used ECG sensor, heartbeat sensor and temperature sensor to monitor the affected person's heart beat rate and pulse rate respectively. Hence similar to using thermometer in house to see blood heat before doctor's consultation, this proposed model (devices) as first-aid data to the patient otherwise now-a- days consulting medical doctors or approaching diagnosis are very high-priced in financial aspect. To reduce this circumstance, we describe the layout of a arduino Uno R3 microcontroller primarily based advanced/high overall performance integrated portable health monitoring system. Like, the heart beat rate (HBR) of the patient is measured by means of placing a finger on the IR led in the heart beat sensor and hence the HBR is displayed on LCD. An SMS will be sent with the help of GSM when the HBR exceeds the threshold. This threshold can be assumed by the programmer while coding. The heart beat ranges among 60 to 100 pulses/min and degree Centigrade ranges from $18^{\circ} \mathrm{C}$ to $38^{\circ} \mathrm{C}$. The body temperature, ECG, HBR is then sent consequently to the authorized person through GSM. 


\section{IoT framework}

Three basic IoT in healthcare framework were taken into account the foremost fundamental thing of IoT in healthcare because it assists in healthcare applications to absolutely make use of cloud computing and IoT. This architecture also supports protocols to protect the communication, program of raw medical motions from several sensors and smart plans for network fog lumps. There are three important components such as structure, topology and platform. Each element supports a specific function within the IoT healthcare framework. This system collects health condition information from various sensor. After receiving the information are transmitted for analyzing through remote server and the real time results obtained will be displayed on an LCD display.

\section{Three basic supplements and their features in the IoT framework for healthcare}

\subsection{IoT Topology}

The IoT topology knob the arrangement of widespread IoT additives and descriptions some trendy setups for given application scenarios within the framework. It represents cloud computing and an IoT in healthcare topology consist of three major elements. First of all, a publisher produce a network of connected sensors or hand-held liable for recording patient's crucial signs, and constantly sending a large amount of raw data like electromyography (EMG), electrocardiogram (ECG), blood sugar (BG), blood heat and therefore the volume of air inhaled and exhaled by lungs to a broker. Following, the broker studies and goods processed data on the cloud. Finally, a subscriber, directly screens patients can access the information from anywhere and responds nearly when any incidents occur.

\subsection{Short-Range Communication Techniques (SRCT)}

Short-range conversation normally functions between tool lumps when treating happens, especially using smart intelligent sensors and gateway/controller. A signal can transit from few centimeters to many meters. Ability of these strategies are frequently carried out for different sort of networks. we only concentrate in the event of a little Medical body area network (MBAN) consists of one central node and a number of other sensors nodes. Among the SRCT, the most extensively used one are Bluetooth, infrared and wi-fi. Important traits of the three strategies are described in supported of a kind feature. Wireless and Bluetooth are typically utilized in cloud computing and IoT for applications of healthcare in wearable sensor.

\subsection{Cloud Computing for Healthcare}

The cloud computing has become one among popular topic in Information technology. It's mobility, scalability and security benefits provides on demand computing resources (e.g., services, storage, servers, networks, hardware etc.,) to users. Nowadays cloud computing has developed as a backbone in IoT healthcare systems. The major benefits of cloud computing is the ability of distributing information among health professionals, caregivers, and patients, 
thus reduces the of medical records lost [20]. Accordingly, healthcare services and packages have enhanced from the event of technologies like Cloud Computing and IoT.

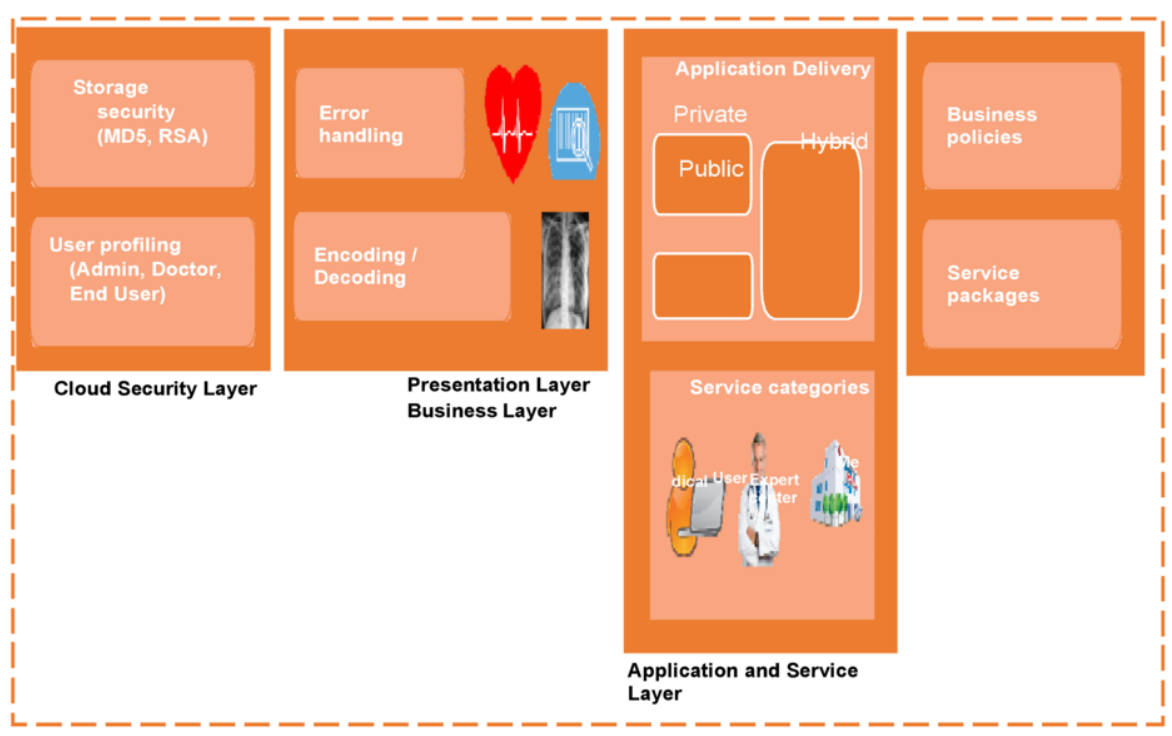

Figure 1. System Architecture for IoT patient health monitoring system using wearable sensor

Divided healthcare applications into two major groups businesses to assists readers to gain better information of this huge topic. The first group address the concept of convergence of cloud computing and IoT within the healthcare, whereas the second group focus on segregating applications of healthcare into two or more distinct group: such as single parameter and multi parameter. Single parameter handles illness and exact disease and multi parameters deals over one disease or an entire. The classification can be altered and enlarged by applying more ideas with different applications or characteristics, including single and multi parameter.

\subsection{Cloud security requirements}

Reliability

Interoperability

Portability

Scalability

Confidentiality 


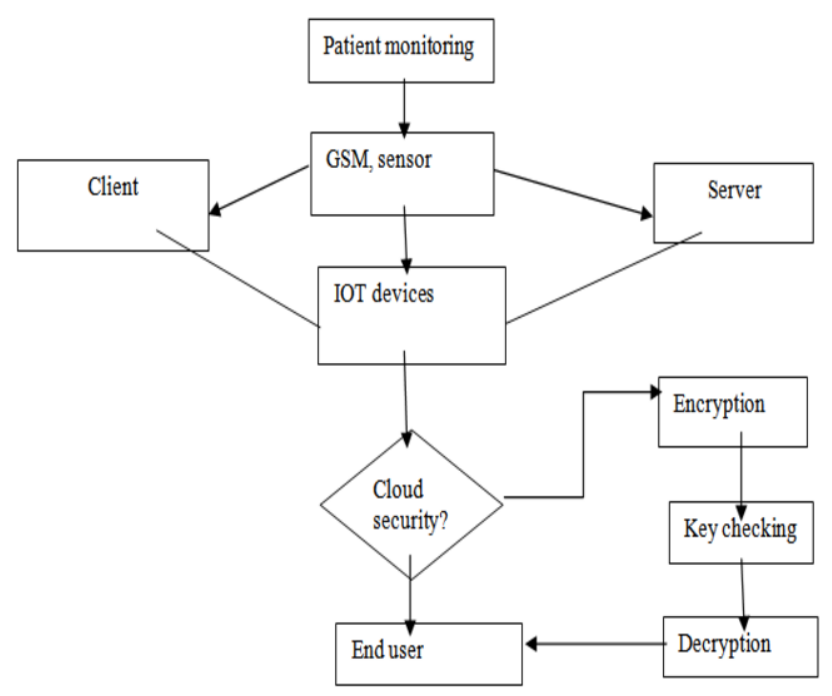

Figure 2. Flow Chart for IoT patient health monitoring system using wearable sensor

\subsection{Fundamental concepts}

The cloud computing and IoT are transfiguring the healthcare industry by introducing various ideas to research area, and each idea supports for various healthcare applications. However, its very tough to represent the general concept of cloud computing and IoT in healthcare. This paper introduce concept as a trending solution have the possibility for an inventory of application and solution. Nowadays, healthcare industries are being working advanced, and new ideas were consistently introduced.

The subsequent sections highlight several basic concepts of cloud computing IoT within the healthcare.

\subsection{LCD Display}

The obtained results in patient monitoring such as ECG, Blood pressure, Heart rate and Temperature will be displayed using an LCD Display. LCD operated with a single $5 \mathrm{~V}$ power supply.

\subsection{MAX 232}

The device consists of dual receiver/ driver which include a capacitive voltage generator to pass voltage level of TIA/EIA-232-F from a 5V supply.The TIA/EIA232-F inputs is converted into 5-V TTL/CMOS levels by each receiver. 


\subsection{GSM}

It is used for communicating data service or transmitting mobile data or voice data. The obtained results in patient monitoring such as ECG, Blood pressure, Heart rate and Temperature will be transmitted to the patient's caretaker using a GSM

\subsection{Power supply}

The input power supplied to the system is $5 \mathrm{~V}$. Output voltages of $5 \mathrm{~V}, 12 \mathrm{~V}$, and $15 \mathrm{~V}$ supply.

\subsection{Temperature Sensor}

Temperature sensor evaluate the amount of heat or coldness in the patient's body. It sense or detects any physical change to that temperature.

\subsection{Heart beat Sensor}

Heart beat rate [10] can be observed by two ways one is by checking the pulse either in neck or wrist and the other way is using heart beat sensor. It determines the condition of patient's heart. Heartbeat Sensor works under the principle of Photoplethysmograph. The normal heart beat rate of adult ranges from 60 to 100 beats per minute.

\subsection{Electrocardiography Sensor}

ECG measures the electrical activity created by heart muscle depolarizations propagates in generating electrical waves towards the skin.The ECG setup contains four electrodes that are pointed at the four extremities (i) $\mathrm{RA}=$ right arm; (ii) LA = left arm; (iii) $\mathrm{RL}=$ right leg; (iv) LL = left legs or on the chest. ECG electrodes are basically a wet sensors and it requires a conductive gel to improve the conductivity among electrodes and skin.

\subsection{Crucial sign}

Vital sign (BP) is that the stress of circulating blood on the walls of blood vessels. Without additional specification, "blood pressure" generally refers to the pressure in large blood vessel of the circulation. Vital sign is generally expressed in terms of the blood strain

\subsection{Eye blink Sensor}

It is based on IR. For every eye blink we can observe the variation in eye. The output is high, when the eyes are closed or else the output will be low when the eyes are open. 
This output is given to logic circuit to indicate the alarm.

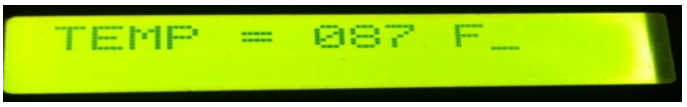

(a)

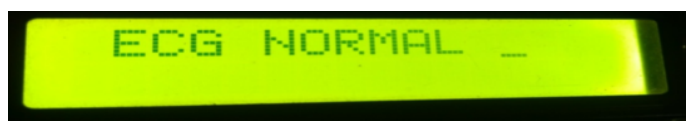

(b)

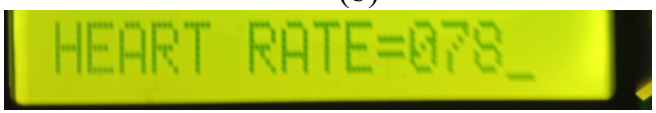

(c)

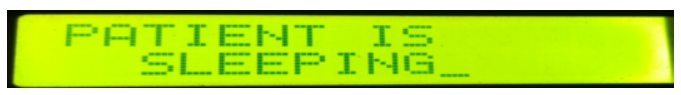

(d)

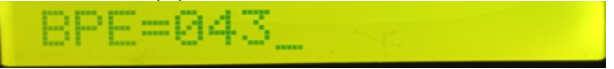

(e)

Figure 3. (a) Output from Temperature sensor (b) Output from ECG sensor (c) Output from Heart beat sensor (d) Output from Eyeblink sensor (e ) Patient's Blood Pressure

Table 1. A wearable Patient Healthcare Monitoring System Using IoT And Cloud Computing based security

\begin{tabular}{|c|c|c|c|c|c|c|}
\hline $\begin{array}{c}\text { PATIEN } \\
\text { T ID }\end{array}$ & HB & ECG & $\begin{array}{c}\text { EYE } \\
\text { BLINK } \\
\text { SENSO } \\
\text { R }\end{array}$ & $\begin{array}{c}\text { B } \\
\text { P }\end{array}$ & $\begin{array}{c}\text { BODY } \\
\text { MOVEME } \\
\text { NT }\end{array}$ & $\begin{array}{c}\text { TEMERATU } \\
\text { RE }\end{array}$ \\
\hline 1. & $\begin{array}{c}07 \\
8\end{array}$ & NORMAL & $\begin{array}{c}\text { SLEEPI } \\
\text { NG }\end{array}$ & $\begin{array}{c}\text { O4 } \\
3\end{array}$ & NO & $087 \mathrm{~F}$ \\
\hline 2. & $\begin{array}{c}08 \\
5\end{array}$ & $\begin{array}{c}\text { ABNORM } \\
\text { AL }\end{array}$ & $\begin{array}{c}\text { RUNNI } \\
\text { NG }\end{array}$ & $\begin{array}{c}06 \\
1\end{array}$ & ACTIVE & $090 \mathrm{~F}$ \\
\hline 3. & 06 & $\begin{array}{c}\text { ABNORM } \\
\text { AL }\end{array}$ & $\begin{array}{c}\text { RUNNI } \\
\text { NG }\end{array}$ & $\begin{array}{c}08 \\
0\end{array}$ & ACTIVE & $093 \mathrm{~F}$ \\
\hline
\end{tabular}




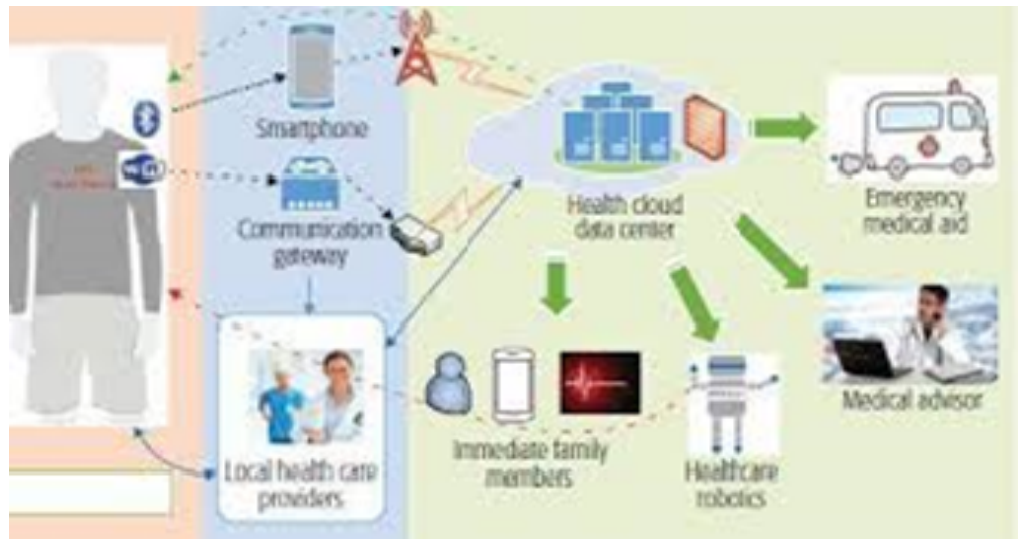

Figure 4. Secure IoT based modern health care system in end -end agreement.

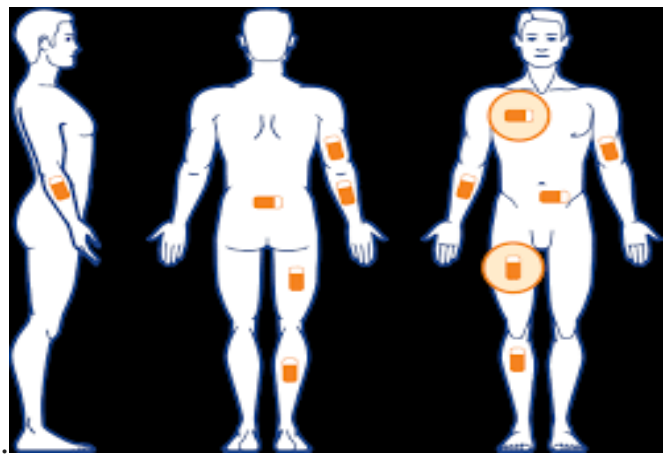

Figure 5. Placement a sensor module
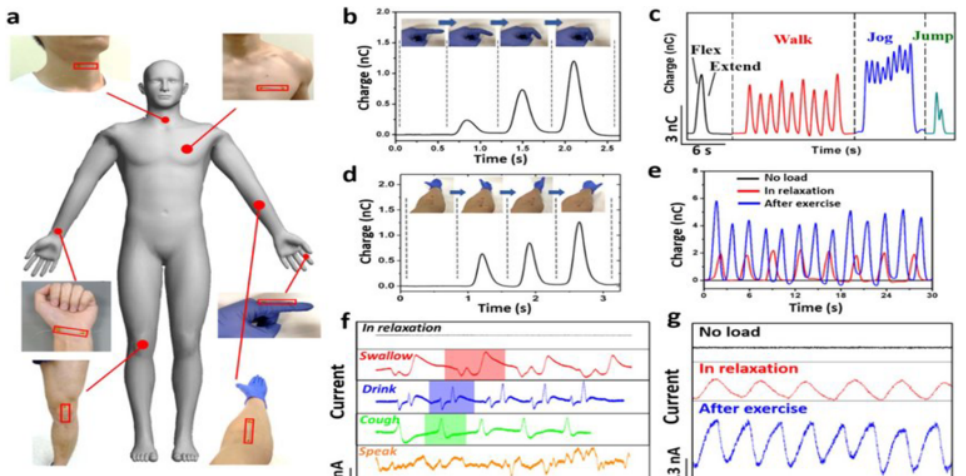

d
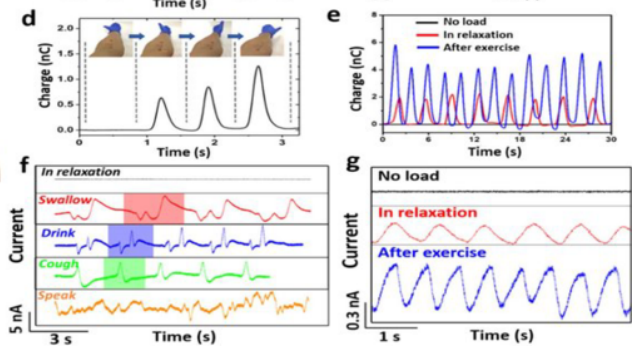

Figure 6. (a ,b ,c) Wearable patient healthcare monitoring system using IoT and cloud computing based security 


\title{
4. Security analysis
}

In the security analysis phase, the cost of the cryptographic hash function for the random parameters $(\mathrm{X}, \mathrm{Y})$ consists of Encryption and Decryption standards with key functions. The cryptographic hash function at the sender side can have patience

At the receiver side

$$
\mathrm{Hs}=\mathrm{h}(\mathrm{X}\|\mathrm{M}\| \mathrm{Ek})
$$

$$
\mathrm{Hr}=\mathrm{h}(\mathrm{Y}|| \mathrm{Dk} \| \mathrm{M})
$$

In our proposed scheme, authentication is done by validating the hash function and random value $\mathrm{X}$ of the patient in the message authentication, where only an authorized sender can send a valid authentication message. The random value $X$, which is calculated from patient id $(\mathrm{X}=\mathrm{PID})$, and a Server id can be obtained by $\mathrm{Y}=(\mathrm{S}$ ID) The overall authenticate the legitimacy of the security techniques in the telemedicine using the hash value 1 and random value $\mathrm{X}$ and $\mathrm{Y}$.

$$
\mathrm{H}=\mathrm{h}(\mathrm{M}\|\mathrm{X}\| \mathrm{Y}\|\mathrm{Ek}\| \mathrm{Dk})
$$

Which must be equal to the hash fee $\mathrm{H}$ (h). By this way, our proposed method satisfies the mutual authentication property. Environments using Java Cryptographic Extension (JCE) [23]] on a smart phone of HTC Desire (as LPU in our proposed machine) with 0.72 GHZ Arm Cortex-A8 CPU and Li-Ion $2000 \mathrm{mAh}$ battery as a test bed .The gsm runs Android 2.2 mobile operating gadget that helps a subset of java center libraries, and in which we also set up JCE. Now, the usage of JCE with the help of the java middle libraries we calculate the execution time and CPU cycles for each 256 -bit block encryption. Here, we take a 1024-bit of records length that's divided into 4 256bit blocks, where each block cipher in hash characteristic takes $6.56 \times 10-1$ execution time and CPU cycle for each 256-bit block encryption of hash characteristic is $9.5 \times$ 10 -four msec. Therefore, for making sure each the privateness and integrity of the facts size of 1024-bit should be taken for calculating the execution time of a CPU machine takes is Execution time of a

$\mathrm{CPU}=(\mathrm{CPU}$ Clock cyclizes + Memory stall cycles $) \times$ Clock cycle time $=(6.56 \times 10-1$ $+0) \times 9.5 \times 10-4=0.0006232 \mathrm{msec}$.

Here there is no memory access per instruction, so it can be as 0 . Whereas, the existing work BSN care can have the CPU clock cycles time of $0.007655 \mathrm{~m} \mathrm{sec}$ [24]. Therefore, our proposed data security approach used causes less than half Computational overhead and execution time as compared to BSN care, which is greatly useful for the resource constrained in IOT sensor devices.

\subsection{Key Management}

\author{
Select the Key index K \\ Key takes Security Parameter $\alpha$ to return secret Key K where K> $>$ \\ Message (M) \\ Encryption $=(\mathrm{M}, \mathrm{D})=$ Cipher Message $(\mathrm{C})$ \\ Decryption $=(\mathrm{C}+\mathrm{M})$
}

Key Management to be handle by set of key constraints (K) which takes security parametera, an intelligible message (M). By applying data encryption and decryption standard we will get sample data to monitor. 


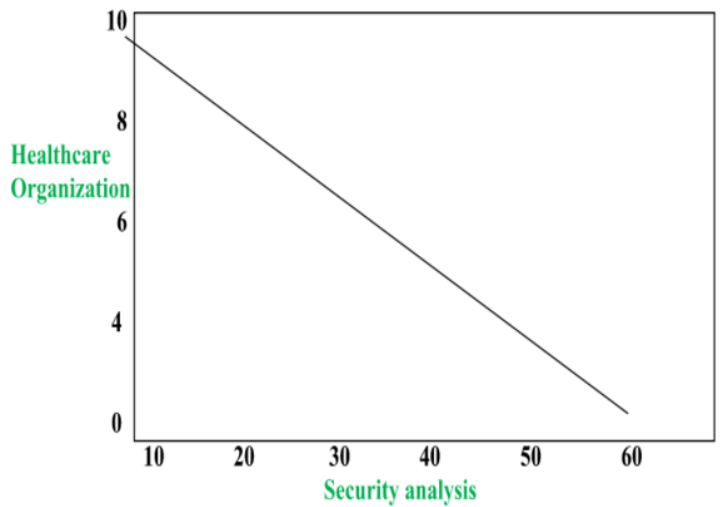

Figure 7. Performance of the system

\section{Conclusion}

In this paper, we have analyzed Microcontroller based complete health monitoring tool using IoT and cloud computing. Any abnormalities within the health stipulations can be acknowledged right away and are knowledgeable to an appropriate individual via wi-fi technological information or through Internet from the collected facts. The proposed device is simple, strength green and handy to understand. It acts as a network between the doctor patient handiest share statistics for the usage of security. The hardware for venture is applied and the results are demonstrated successfully.

\section{References}

[1] R.L.Bashshur, T.G., AND G.W.shannon .telemedicine: a new health care delivery system.ann.rev.public ealth,vol.21,2000,pp.613-17.

[2] Bayat ,M,Barmshoory,M,rahini,M,\&aref,M.R(2015).secure authentication scheme forVANETS with batch verification .wireless network ,21(50,1733-1743).

[3] Azees, maria.etc al.'EAAP: efficient anonymous authentication with conditional privacy preserving scheme for vehicular adhoc network. IEEE transactions oninteligent transportationsystem, vol18, no9, 2017,pp.2467-2467.

[4] Sha,M.identity-based encryption with outsourced equality test in cloud computing.inf,sci.2016, 328,389-402.

[5] Pairing based cryptography library 9online) Available:http://crypto.standard.edu/pub/.

[6] Y.kong,M.zhang, and.ye .A belief prorogation -based method for task allocation in open and dynamic cloud environment. knowledgebase system, vol115,2016,pp.123-32.

[7] G.Hackmann et al..Cyberphysical codesign of distribution structural health monitoring with wieless sensor network .IEEE trans.parallel and distrib.system ,vol.24,no,1,2014,pp.63-72.

[8] Azzes,M,vijayakumar,P.,\&deboarth,L.J(2017).EAAP;Efficient anonymous authentication with conditional privacy - preserving scheme for vehicular ad hoc network.IEEE transaction on intelligent transportation system.

[9] S.Hema Kumar, J.Uday Kiran, V.D.Ambeth Kumar, G.Saranya, Ramalakshmi V, "Effective Online Medical Appointment System.International Journal of Scientific \& Technology Research, Volume 8, Issue 09, September 2019, Pages $803-805$.

[10] V.D.Ambeth Kumar, G.Gokul, S.Malathi, K.Vengatesan, D.Elangovan, B.Chitra, "Implementation Of The Pulse Rhythemic Rate For The Efficient Diagonising Of The Heart Beat", ", Healthcare Technology Letters (IET) 2019 Apr 17;6(2):48-52.

[11] V.D.Ambeth Kumar, G.Gokul, S.Malathi, K.Vengatesan, D.Elangovan, B.Chitra, "Implementation Of The Pulse Rhythemic Rate For The Efficient Diagonising Of The Heart Beat", ”, Healthcare Technology Letters (IET) 2019 Apr 17;6(2):48-52.

[12] V.D.Ambeth Kumar (2018), “ NOVEL WIRELESS SENSING SYSTEM FOR THE WELFARE OF SEWER LABORERS”, Healthcare Technology Letters (IET) Volume 5, Issue 4, p. 107 -112 